\title{
ISSN $2237-048 X$

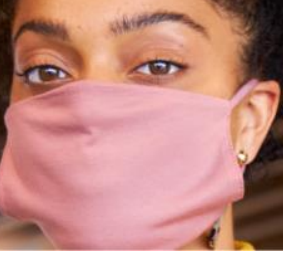 \\ RELAÇÃO ENTRE O CONSUMO DE ÁLCOOL E A QUALIDADE DO SONO COM O RISCO CARDIOVASCULAR EM JOVENS SAUDÁVEIS
}

MARTINS, D. A. W¹; BACK, G. D1; HWAN, C.-L², TRIMER, R³; SILVA, A. L. G³; PHILLIPS, S. A².

PALAVRAS-CHAVE: Transtornos relacionados ao uso de álcool. Risco vascular. Fatores de risco. Sono.

\begin{abstract}
RESUMO
Introdução: $O$ consumo excessivo de bebida alcoólica (BA) é um sério problema de saúde que está associado ao desalinhamento do sono, da função cardiovascular e aumento da mortalidade. Objetivo: Avaliar relação entre BA e qualidade do sono (QS) com o risco cardiovascular em jovens saudáveis. Métodos: Recorte transversal da pesquisa "Binge drinking and cardiovascular health in young adults" da Universidade de Illinois de Chicago. Recrutados 48 sujeitos que, após avaliação clínica, foram estratificados conforme o consumo de álcool em 03 grupos: "Bebedores Abstinentes" (AB; $n=11$ ); "Bebedores Moderados" (BM; $n=17)$; "Bebedores Compulsivos" (BC; $\mathrm{n}=18$ ). QS foi avaliada pelo Questionário de Pittsburgh e classificada em Boa ou Ruim. 0 risco cardiovascular foi avaliado pela Velocidade da Onda de Pulso (VOP) e Pressão de Pulso Central (PPcentral) por tonometria de aplanação e um transdutor de alta fidelidade de tensão de calibre. Os resultados foram analisados por meio da correlação de Spearman e considerado significativo $p<0,05$. Resultados: A PPcentral foi mais elevada no BC, comparado ao $B M(33,0 \pm 5,3$ vs $28,9 \pm 3,5, p=0,034)$, a VOP apresentou tendência a elevação em $B C(5,1 \pm 0,5)$ e BM $(5,1 \pm 0,6)$ comparado aos $A B(4,6 \pm 0,5)$. QS (escore total e componentes) foi semelhante entre os grupos. Apenas sujeitos com QS Ruim: $B C(n=11)$ apresentaram maior PPcentral comparado ao $B M(n=10)(34,5 \pm 5,8$ vs $29,2 \pm 3,9, p=0,046)$. BA correlacionou-se com os distúrbios do sono $(r=0,310, p=0,036)$ e com a PPcentral $(r=0,336, p=0,012)$. Conclusão: Jovens adultos $B C$ apresentam mais elevada PPcentral e tendem a maior rigidez arterial pela VOP. Distúrbios do sono e PPcentral estão relacionados ao consumo de BA.
\end{abstract}

\section{RELATIONSHIP BETWEEN ALCOHOL CONSUMPTION AND SLEEP QUALITY WITH CARDIOVASCULAR RISK IN HEALTHY YOUNG PEOPLE}

KEYWORDS: Disorders related to alcohol use. Vascular risk. Risk factors. Sleep.

\begin{abstract}
Introduction: Excessive consumption of alcoholic beverages $(A B)$ is a serious health problem that is associated with misalignment of sleep, cardiovascular function and increased mortality. Objective: To evaluate the relationship between $\mathrm{AB}$ and sleep of quality (SQ) with cardiovascular risk in healthy young people. Methods: Crosssectional survey of the "Binge drinking and cardiovascular health in young adults" research at the University of Illinois at Chicago. After clinical evaluation, 48 subjects were recruited and stratified according to alcohol consumption in 03 groups: "Alcohol Abstainers" (AA; $n=11)$; "Moderate Drinkers" (MD; n=17); "Binge Drinkers" (BD; $n=18$ ). SQ was assessed by the Pittsburgh Questionnaire and classified as Good or Bad. Cardiovascular risk was assessed by Pulse Wave Velocity (PWV) and central Pulse Pressure (cPP) by applanation tonometry and a high-fidelity gauge voltage transducer. The results were analyzed using Spearman's correlation and considered significant $p<0,05$. Results: $c P P$ was higher in BD compared to MD (33.0 \pm 5.3 vs $28.9 \pm 3.5, p=0.034)$, PWV tended to increase in BD $(5.1 \pm 0,5)$ and MD $(5.1 \pm 0.6)$ compared to AA $(4.6 \pm 0.5)$. SQ (total score and components) was similar between groups. Only subjects with poor SQ: BD $(n=11)$ had a higher PPcentral compared to MD ( $n=10)$ (34.5 \pm 5.8 vs $29.2 \pm 3.9, p=0.046)$. AB correlated with sleep disorders $(r=0.310, p=0.036)$ and with $c P P(r=0.336$, ${ }^{1}$ Acadêmicos de Fisioterapia, Universidade de Santa Cruz do SuI - UNISC. E-mails: douglasmartins8@hotmail.com,
\end{abstract}

guilhermeback4@gmail.com.

${ }_{2}^{2}$ Departamento de Fisioterapia, Universidade de Illinois de Chicago - UIC. E-mail: clhwang@uic.edu, shanep@uic.edu.

${ }^{3}$ Docente do Departamento de Ciências da Saúde, Universidade de Santa Cruz do Sul - UNISC E-mail: andreag@unisc.br, renatatrimer@unisc.br. 
$\mathrm{p}=0.012)$. Conclusion: Young BD adults have higher cPP and tend to have higher arterial stiffness due to PWV. Sleep disorders and cPP are related to the consumption of $A B$.

\section{INTRODUÇÃO}

O uso crescente de bebida alcoólica no meio universitário é alarmante. Homens e mulheres estudantes universitários consomem bebidas alcoólicas (BA) em média 10 dias por mês, de 4-6 drinques por episódio (WUNDT et al., 2009). No Brasil, segundo dados do PNS-IBGE (2013), a idade de início do consumo de BA foi 18,7 anos, sendo mais prevalente na Região Sul com $28,4 \%$ de consumidores, variando conforme o sexo. 0 consumo de BA geralmente se inicia na adolescência (HASLER et al., 2015) e está envolvido com graves alterações na arquitetura do sono (WILKINSON et al., 2018), como a perturbação do sono (dificuldade em adormecer), duração insuficiente do sono (privação ou restrição do sono) e hipersonia (o desejo excessivo e/ou necessário) (HASLER et al., 2015). Nos Estados Unidos, um estudo representativo nacional documentou que $80,7 \%$ dos estudantes universitários já havia consumido bebida alcoólica e destes, mais da metade (55,5\%) se envolveram em episódios pesados de consumo de álcool e foram responsáveis pela grande maioria do consumo geral de álcool no campus universitário (WECHSLER et al., 2002). Os resultados documentaram altas taxas de sofrimento mental, má qualidade global do sono e ocorrência de problemas de saúde mental-sono-álcool nesta grande amostra de estudantes universitários considerados bebedores compulsivos (KENNEY et al., 2013).

Embora haja um aumento na compreensão do público e dos esforços de prevenção e intervenção com o intuito de reduzir o consumo problemático de bebidas alcoólicas, as taxas de prevalência de consumo de alto risco e consequências relacionadas permanecem desde muitos anos (KENNEY et al., 2012). Em adultos jovens, este hábito frequente está ligado à disfunção vascular que prepara o cenário para doenças crônicas e eventos cardiovasculares (CV) posteriores (REHM et al., 2013; SUNDELL et al., 2008), como a hipertensão arterial sistêmica (HAS), aterosclerose carotídea, acidente vascular cerebral, infarto e morte súbita (JOHNSTON et al., 2013; MUKAMAL et al., 2005; WUNDT et al,. 2009). Entretanto, o real impacto do uso contínuo de BA sobre a saúde cardiovascular dos jovens ainda carece de esclarecimentos mesmo se tratando de um vício que fere gravemente a saúde dos consumidores. Dessa forma, se faz imperativo conhecer as alterações que o uso recorrente de BA provoca na saúde de uma maneira geral e, nesse sentido, buscamos avaliar a relação entre o consumo de álcool sobre a qualidade do sono e risco cardiovascular em jovens saudáveis.

\section{FUNDAMENTAÇÃO TEÓRICA}

O consumo excessivo de álcool é um dos mais graves problemas de saúde pública que as faculdades americanas enfrentam (GOSLAWSKI et al., 2013). Estudos demonstram que problemas no sono previram prospectivamente o uso indevido de álcool em amostras da comunidade, recaída entre alcoólatras e consumo de álcool entre adolescentes com o início precoce entre 12 e 14 anos (KENNEY et al., 2013). A necessidade de doses cada vez mais elevadas de álcool, à medida que os consumidores aumentam sua tolerância ao químico, está associada aos riscos relacionados ao sono induzido por álcool. 0 sono fragmentado e ineficiente resulta em um tempo menor de descanso que afeta negativamente na qualidade do sono (KENNEY et al., 2012). 
0 consumo desenfreado de BA está associado com resultados diretos sobre o aumento no risco de eventos cardiovasculares em adultos com idades entre 40 e 60 anos (GOSLAWSKI et al., 2013). Conforme a Organização Mundial da Saúde, as doenças cardiovasculares são a principal causa de morte no mundo chegando a 17,7 milhões de óbitos por doenças cardiovasculares em 2015 , o que representa $31 \%$ de todas as mortes em nível global (OPAS/OMS, 2017). Já no Brasil, estima-se que cerca de 600 milhões de pessoas tenham HAS e ocorram 7,1 milhões de mortes anuais decorrentes dessa doença, com um crescimento mundial de $60 \%$ de novos casos até 2025 (MS, 2018).

Em adultos, o consumo de BA está associado a elevação da pressão arterial (PA), que predispõe a inflamação vascular e contribui para lesões em determinados órgãos, como o sistema nervoso central (PARATI et al., 2002). Variações da PA muitas vezes são despercebidas (MUSINI et al., 2009), e esta, sofre flutuações constantes em um período de 24 horas. Com o monitoramento ambulatorial não invasivo da PA (MAPA) é possível avaliar as variações da PA como um preditor de risco cardiovascular (RCV) e indicar significativas flutuações diárias (DIAZ et al., 2013). Desta forma, critérios de controle ambulatorial da PA têm se definido com um importante preditor de RCV do que as medidas de PA no consultório médico (CLEMENT et al., 2003; DOLAN et al., 2005).

Nesse sentido, diversos mecanismos podem estar interligados ao aumento do risco de eventos cardiovasculares adversos; no entanto, um mecanismo central pode ser alterações na biologia vascular, como disfunção endotelial (GOSLAWSKI et al., 2013). Dentre as avaliações diagnósticas existentes, a velocidade da onda de pulso (VOP) serve como um marcador para o enrijecimento arterial, bem como Pressão de Pulso Central (PPcentral) (MOON et al., 2018).

\section{MATERIAIS E MÉTODOS}

\subsection{Delineamento do estudo}

Estudo de delineamento transversal, com amostragem de conveniência, realizado junto ao banco de dados do projeto de pesquisa intitulado "Binge drinking and cardiovascular health in young adults" na Universidade Illinois at Chicago - UIC, Chicago - EUA, devidamente aprovada pelo Comitê de Ética em Pesquisa desta universidade (Protocol \#2017-0305), na qual todos os sujeitos consentiram participar da pesquisa mediante assinatura do Termo de Consentimento Livre e Esclarecido.

Foram incluídos no estudo 46 indivíduos adultos jovens (18-30 anos), sem doença cardiovascular, por conveniência recrutados da comunidade e campus universitário da UIC, sendo excluídos aqueles que tivessem algum conhecimento sobre possuir fatores de risco cardiovascular (histórico de tabagismo, diabetes, doença renal, distúrbio convulsivo, câncer, inflamação ativa), doença metabólica, gravidez e uso de drogas ilícitas.

\subsection{Mensurações}

Os pacientes foram submetidos inicialmente a avaliação clínica para levantamento de dados sociodemográficos e estratificados conforme o consumo de álcool em três grupos por meio do Questionário Alcohol Use Disorders Identification Test (AUDIT): "Bebedores Abstinentes" (AB; $n=11)$, "Bebedores Moderados" 
(BM; n=17), "Bebedores Compulsivos" (BC; $n=18)$ (FIGURA 1). Posteriormente, os indivíduos foram submetidos a análise da Qualidade do Sono (QS), por meio do Questionário de Pittsburgh e avaliação do Risco Cardiovascular pela coleta da Velocidade da Onda de Pulso (VOP) e Pressão de Pulso Central (PPcentral).

\subsection{Consumo de Álcool (CA)}

O consumo de álcool foi avaliado através do Questionário Alcohol Use Disorders Identification Test (AUDIT), sendo classificado como consumo de baixo risco (0-7 pontos) à provável dependência (20 ou mais pontos). Nesse sentido, os pacientes foram estratificados em três grupos: "Bebedores Abstinentes" (AB), definidos como aqueles que não consomem mais que 1 BA padrão por mês, nos últimos 2 a 3 anos; "Bebedores Moderados" (BM), para homens, consumo de não mais de 3 bebidas padrão por vez com frequência máxima de 1-2 vezes por semana e para mulheres o consumo de não mais de 2 bebidas padrões por vez com não mais de 1-2 vezes na semana nos últimos cinco anos; "Bebedores Compulsivos" (BC) definidos como 5 ou mais bebidas em um período de 2 horas nos últimos 30 dias se homem e 4 ou mais no período de 2 horas para mulheres.

\subsection{Qualidade do Sono (QS)}

A qualidade do sono foi avaliada através do Questionário Pittsburgh, sendo classificado em boa qualidade do sono (0 e 4 pontos) ou ruim qualidade do sono (5 e 10 pontos), uma vez que acima de 10 pontos é considerado a presença de distúrbio do sono. 0 questionário é dividido em 7 componentes: 1-Qualidade subjetiva do sono, 2Latência do sono, 3-Duração do sono, 4-Eficiência habitual do sono, 5-Distúrbios do sono, 6-Uso de medicação para dormir e, 7-Disfunção durante o dia.

\subsection{Risco Cardiovascular (RCV)}

O risco cardiovascular foi avaliado por meio do SphygmoCor XCEL (SphygmoCor; AtCor Medical, Austrália), onde foram obtidas a Velocidade da Onda de Pulso (VOP) e Pressão de Pulso Central (PPCentral). Para isso, os pacientes foram orientados a permanecer em decúbito dorsal durante 10 segundos utilizando tonometria de aplanação e um transdutor de alta fidelidade de tensão de calibre. A VOP representa a razão da distância entre dois pontos do sistema arterial e o tempo gasto pela onda para percorrer essa distância. Já a PPcentral se caracteriza pela diferença entre a pressão sistólica (Psist) e a pressão diastólica (Pdiast), onde a Psist na aorta central é menor e aumenta à medida que a onda de pulso é transmitida para a periferia e já a Pdiast aumenta menos ou pode até diminuir (MENDES-PINTO et al., 2019).

FIGURA 1. Fluxograma de amostragem e fases do estudo. AUDIT: alcohol use disorders identification test; AB: bebedores abstinentes; BM: bebedores moderados; BC: bebedores compulsivos; VOP: velocidade da onda de pulso; PPcentral: pressão de pulso central; QS: qualidade do sono. 


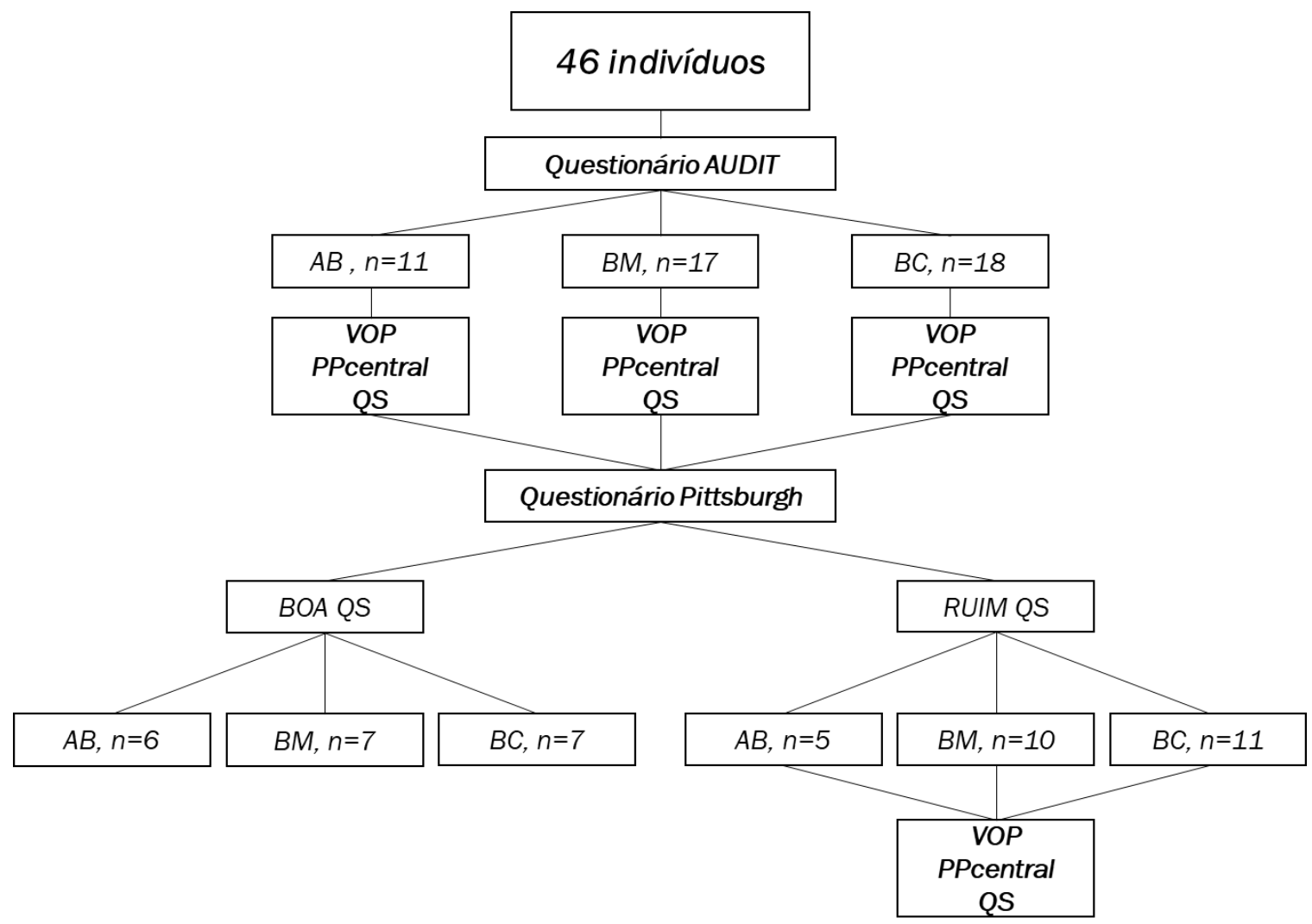

\subsection{Análise Estatística}

Os resultados foram analisados no programa SPSS versão 24.0, expressos em média e desvio padrão ou intervalo de confiança de 95\%. Para análise entre os grupos foi utilizado ANOVA one-way e as associações foram analisadas através da correlação de Spearman, considerando significativo um $p<0,05$.

\section{RESULTADOS}

Nenhuma diferença significativa foi encontrada entre os grupos AB, BM e BC para as variáveis clínicas idade, sexo, índice de massa corporal (IMC) e circunferência da cintura (TABELA I). Em contrapartida, a circunferência do quadril foi maior no grupo $B C$, comparado ao grupo $A B(95,4 \pm 5,6$ vs $89,2 \pm 6,4, p=0,031)$. Durante o repouso, a pressão braquial sistólica e a pressão braquial diastólica não diferiram entre os grupos, porém a VOP apresentou tendência a elevação em BC $(5,1 \pm 0,5)$ e BM $(5,1 \pm 0,6)$ comparado aos $A B(4,6 \pm 0,5)$, sem significância. A PPcentral foi maior no BC, comparado ao BM $(33,0 \pm 5,3$ vs $28,9 \pm 3,5, p=0,034)$.

Tabela I. Consumo de álcool, características clínicas e arteriais em jovens adultos.

\begin{tabular}{lccc}
\hline Grupos & $\begin{array}{c}\text { Abstinentes } \\
(n=11)\end{array}$ & $\begin{array}{c}\text { Moderados } \\
(n=17)\end{array}$ & $\begin{array}{c}\text { Compulsivos } \\
(n=18)\end{array}$ \\
\hline Variáveis & & & \\
\hline
\end{tabular}




\begin{tabular}{lcccc}
\hline Idade (anos) & $21.9 \pm 3.6$ & $24.3 \pm 2.1$ & $23.7 \pm 2.5$ & $N S$ \\
Sexo, Masculino $n(\%)$ & $7(64.0)$ & $9(50.0)$ & $9(50.0)$ & - \\
Raça & - & - & $2(11.0)$ & - \\
Afro-descendentes & $1(9.0)$ & $4(22.0)$ & $3(17.0)$ & - \\
Asiáticos & $4(36.0)$ & $9(50.0)$ & $1(5.0)$ & - \\
Hispânico & $1(9.0)$ & $2(11.0)$ & $12(67.0)$ & - \\
Brancos & $5(46.0)$ & $2(11.0)$ & - & $N S$ \\
Outros & - & $1(6.0)$ & $23.7 \pm 2.5$ & $N S$ \\
IMC $\left(\mathrm{kg} / \mathrm{m}^{2}\right)$ & $21.9 \pm 2.9$ & $23.7 \pm 2.6$ & $81.0 \pm 6.5$ & 0.034 \\
CC $(\mathrm{cm})$ & $76.9 \pm 9.4$ & $82.1 \pm 7.9$ & $95.4 \pm 5.6 *$ & $\mathrm{NS}$ \\
CQ $(\mathrm{cm})$ & $89.2 \pm 6.4$ & $91.4 \pm 6.7$ & $114.52 \pm 8.66$ & $\mathrm{NS}$ \\
PAS $(\mathrm{mmHg})$ & $113.13 \pm 11.25$ & $114.34 \pm 8.94$ & $66.50 \pm 7.57$ & $\mathrm{NS}$ \\
PAD $(\mathrm{mmHg})$ & $69.00 \pm 9.91$ & $65.77 \pm 8.73$ & $5.15 \pm 0.57$ & 0.018 \\
VOP $(\mathrm{m} / \mathrm{s})$ & $4.63 \pm 0.56$ & $5.13 \pm 0.61$ & $33.05 \pm 5.34 \&$ & \\
PPcentral $(\mathrm{mmHg})$ & $30.30 \pm 4.74$ & $28.73 \pm 3.51$ & & \\
\hline
\end{tabular}

Os dados são apresentados como média \pm desvio padrão; $n$ (\%): número de pacientes (\% do tamanho da amostra); IMC: índice de massa corporal; CC: circunferência da cintura; CQ: circunfência do quadril; PAS: pressão arterial sistólica; PAD: pressão arterial diastólica; VOP: velocidade da onda de pulso; PPcentral: pressão de pulso central.

*Compulsivos vs Abstinentes

\#Moderados vs Abstinentes

\&Compulsivos vs Moderados

Na avaliação da Qualidade do Sono pelo Questionário Pittsburgh, observamos uma média no escore total semelhante entre os grupos (TABELA II). Nenhuma diferença significativa entre os grupos foi observada na QS, escore total e seus componentes.

Tabela II. Qualidade do sono e consumo de álcool em jovens adultos.

\begin{tabular}{|c|c|c|c|c|}
\hline Grupos & $\begin{array}{c}\text { Abstinentes } \\
(n=11)\end{array}$ & $\begin{array}{c}\text { Moderados } \\
\quad(n=17)\end{array}$ & $\begin{array}{c}\text { Compulsivos } \\
(n=18)\end{array}$ & Valor $p$ \\
\hline \multicolumn{5}{|l|}{ Variáveis } \\
\hline Escore total PSQI (pontos) & 4.7 (IC: 3.3; 6.0) & 5.6 (IC: 4.1; 7.1) & 5.3 (IC:4.2; 6.4) & NS \\
\hline C1_Qualidade subjetiva do sono & 0.5 (IC:0.08;1.0) & 0.9 (IC:0.6; 1.2) & 0.8 (IC:0.5; 1.1) & NS \\
\hline C2_Latência do sono & 1.0 (IC:0.4; 1.7) & 0.8 (IC:0.3; 1.3) & 1.0 (IC:0.6;1.4) & NS \\
\hline C3_Duração do sono & 0.7 (IC:0.1; 1.2) & 0.9 (IC:0.5; 1.3) & 0.7 (IC:0.3;1.0) & NS \\
\hline C4_Eficiência do sono & 0.09 (IC:-0.1;0.2) & 0.5 (IC:0.1; 0.9) & 0.3 (IC: $-0.008 ; 0.06)$ & NS \\
\hline C5_Distúrbios do sono & 1.0 (IC:0.7; 1.3) & 1.1 (IC:0.9; 1.2) & 1,2 (IC:1.0; 1.4) & NS \\
\hline C6_Uso de medicação para dormir & 0.1 (IC:-0.2; 0.5) & 0.2 (IC:-0.1; 0.6) & 0.05 (IC:-0.06; 0.1) & NS \\
\hline C7_Disfunção diurnal & 1.0 (IC:0.6; 1.5) & 1.0 (IC:0.6; 1.3) & 1.1 (IC:0.7; 1.4) & NS \\
\hline Pontuação de corte de QS ( $\geqslant 5)$ & - & - & - & - \\
\hline Ruim qualidade do sono, $n$ (\%) & $5(45.0)$ & $10(58.8)$ & $11(61.1)$ & NS \\
\hline $\operatorname{VOP}(\mathrm{m} / \mathrm{s})$ & 4.3 (IC:3.7; 4.9) & 5.1 (IC:4.6; 5.6) & 5.1 (IC: 4.6; 5.5) & NS \\
\hline PPcentral (mmHg) & 29.5 (IC:24,1;34,8) & 29.2 (IC:26,3;32,0) & 34.5 (IC:30,5;38,4)\& & 0.046 \\
\hline Consumo de álcool (pontos) & - & - & - & - \\
\hline AUDIT total & 0.8 (IC:-0.3;2.0) & $3.6(I C: 2.5 ; 4.7)^{\#}$ & 7.9 (IC:5.9;9.9)* \& & 0.001 \\
\hline
\end{tabular}

Os dados são apresentados como média \pm desvio padrão; $n$ (\%): número de pacientes (\% do tamanho da amostra); PSQI: questionário do índice de qualidade do sono de pittsburgh; QS: qualidade do sono; VOP: velocidade da onda de pulso; PPcentral: pressão de pulso central; AUDIT: alcohol use disorders identification test.

*Compulsivos vs Abstinentes

\#Moderados vs Abstinentes

\&Compulsivos vs Moderados 
Considerando o ponto de corte $\geq 5$ no escore total do Questionário Pittsburgh para determinar alteração na qualidade do sono, estratificamos no grupo $A B(n=5)$ indivíduos, no grupo $B M(n=10)$ indivíduos e no grupo $B C$ ( $n=11)$ indivíduos apresentando ruim qualidade do sono. Apenas nestes indivíduos, o grupo BC apresentou maior PPcentral comparado ao BM $(34,5 \pm 5,8$ vs $29,2 \pm 3,9, p=0,046)$. Correlações positivas foram encontradas entre o consumo de álcool com o componente distúrbios do sono ( $r=0,310, p=0,036)$ (FIGURA 2) e com a PPcentral $(r=0,336, p=0,012)$ (FIGURA 3).

Figura 2. Associação entre o Componente Distúrbios do Sono do Questionário de Pittsburgh com o Consumo de Álcool do Questionário AUDIT para todos os indivíduos da pesquisa.

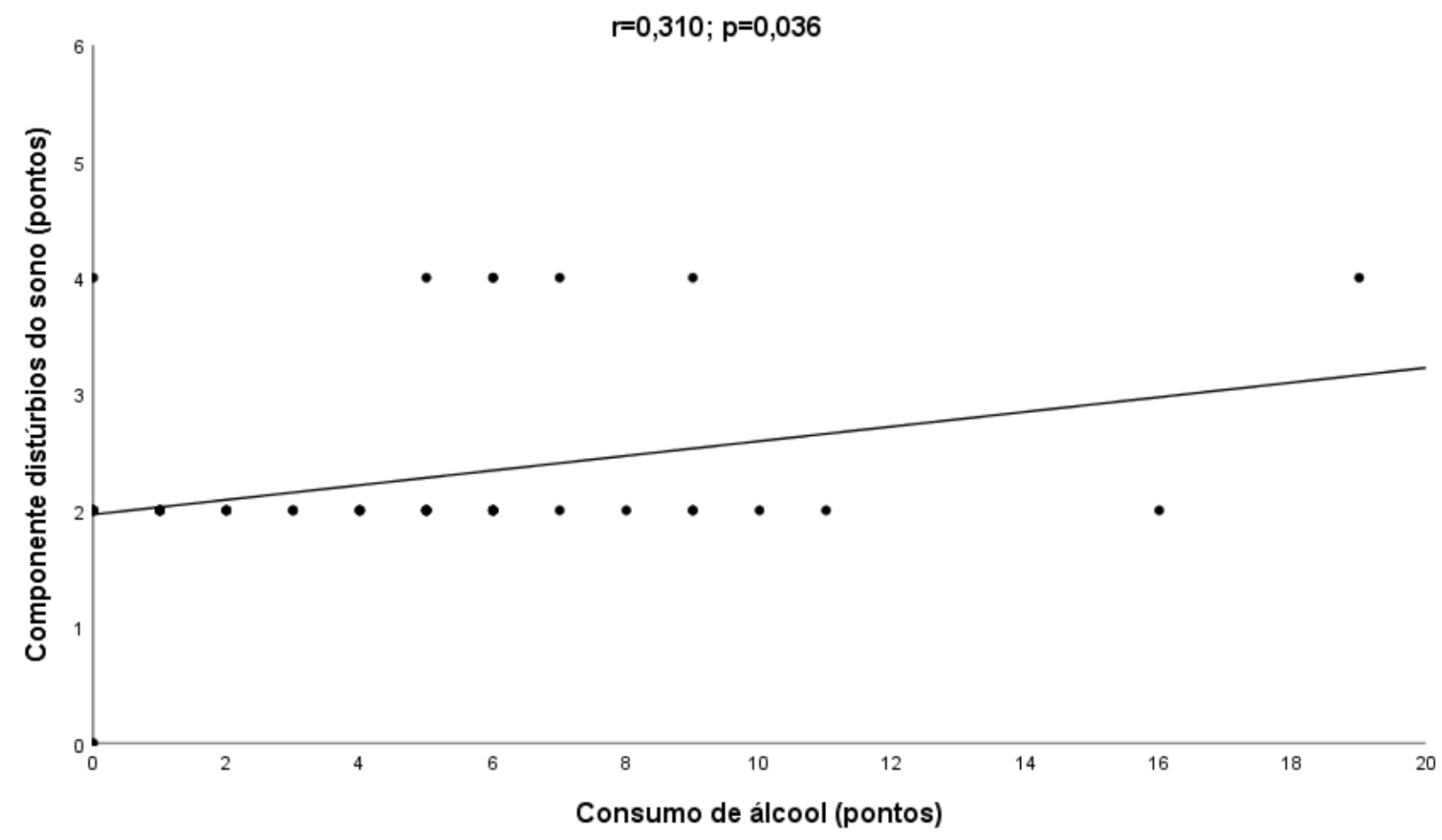


Figura 3. Associação entre a PPcentral com o Consumo de Álcool do Questionário AUDIT para todos os indivíduos da pesquisa.

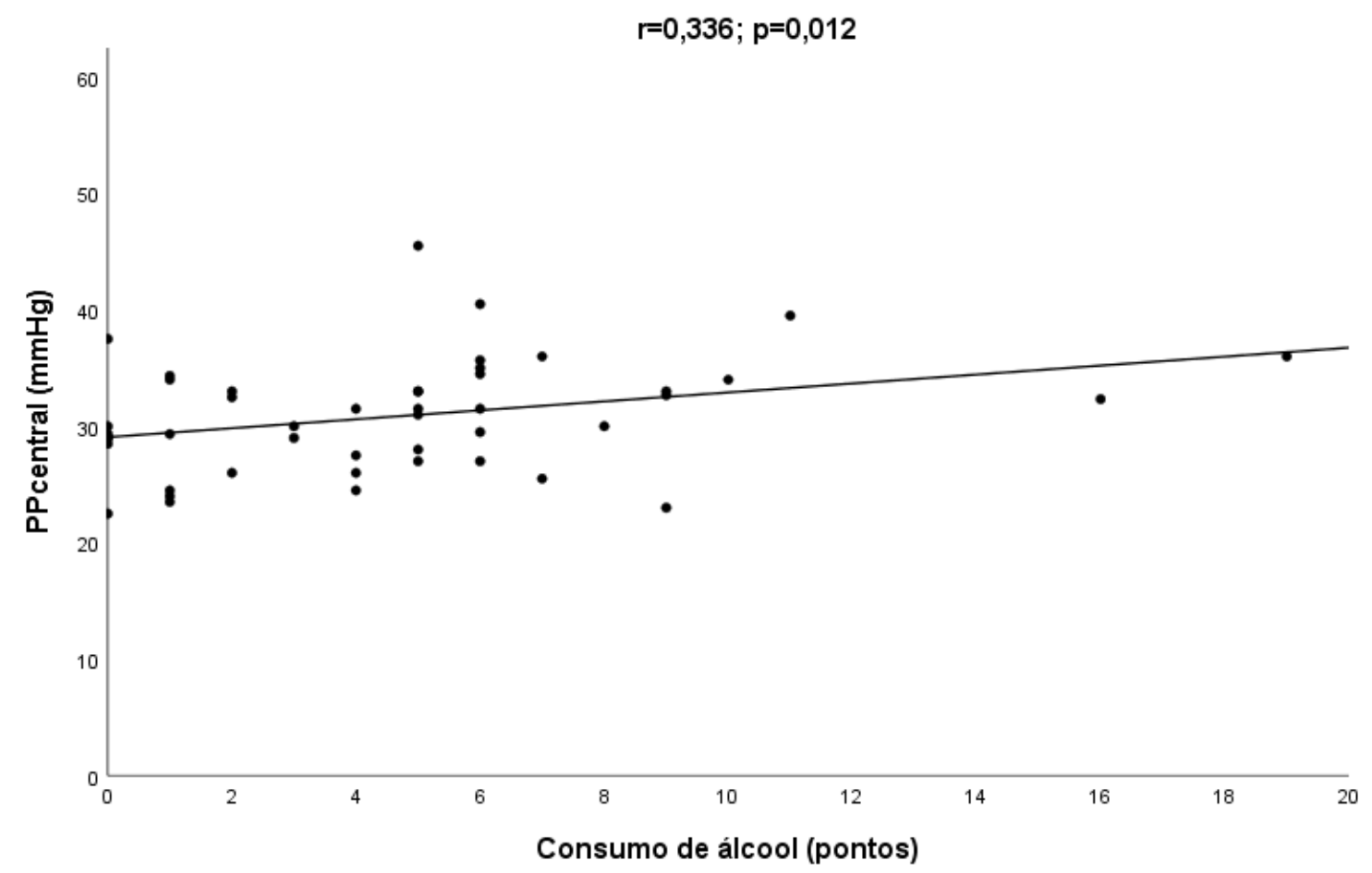

\section{DISCUSSÃO}

Como principal achado deste estudo, podemos destacar que: a) durante o repouso, a VOP apresentou tendência a elevação em $B C(5,1 \pm 0,5)$ e $B M(5,1 \pm 0,6)$ comparado aos $A B(4,6 \pm 0,5)$; b) a PPcentral foi maior no $\mathrm{BC}$, comparado ao BM $(33,0 \pm 5,3$ vs $28,9 \pm 3,5, p=0,034)$. Mendes-Pinto (2019) relataram que o aumento da rigidez arterial avaliada pelo aumento da VOP e PPcentral está associado ao aumento do risco de eventos cardiovasculares, como doença coronariana, acidente vascular cerebral e doença renal terminal (MENDES-PINTO et al., 2019). Hwang e colaboradores (2019) observaram que jovens adultos saudáveis BC e BM apresentaram maior rigidez arterial aórtica comparado aos abstinentes de bebida de álcool, respaldando os nossos achados no que se refere a uma tendência a elevação da VOP em BC e BM e a uma maior PPcentral em BC.

Evidências existentes indicam que o consumo de álcool é responsável pela perturbação do sono, provocando efeitos ainda mais negativos em adolescentes e, ao mesmo tempo, interrompendo a capacidade do sistema circadiano de se adaptar às mudanças de horários (HASLER et al., 2015). Portanto, este achado nos diz que o uso regular de álcool pode resultar em distúrbios do sono como a própria privação do sono, reforçando os achados do nosso estudo, em que indivíduos consumidores de BA podem apresentar correlação positiva com o componente distúrbios do sono (FIGURA 2). 
Nos indivíduos classificados com ruim qualidade do sono, encontramos que o grupo BC apresentou maior PPcentral comparado ao BM $(34,5 \pm 5,8$ vs $29,2 \pm 3,9, p=0,046)$ ". Yaya e colaboradores (2018) relataram que os sujeitos consumidores de BA tiveram chances menores de dormir, por pelo menos 7 horas, quando comparado aqueles que não bebem. Essa privação do sono pode provocar um efeito ativador no sistema de estresse autonômico elevando a frequência cardíaca e a pressão arterial (MEZICK et al., 2014) e isto, pode justificar os nossos achados no que se refere a associação entre a ruim QS e a maior PPcentral nos indivíduos BC, comparado aos indivíduos BM com ruim QS.

Nosso estudo teve várias limitações. Para isolar o efeito do álcool na rigidez arterial, incluímos adultos jovens saudáveis sem presença de fatores de risco cardiovascular tradicionais, o que pode limitar a generalização do estudo. Características demográficas e métricas cardiovasculares não foram diferentes entre os grupos, exceto pela circunferência do quadril, que de forma individual não pode ser considerada um preditor de doenças cardiovasculares. Nosso desenho de pesquisa foi transversal, não permitindo a determinação da causa e efeito, bem como nosso estudo pode não estar equipado para detectar diferenças sexuais no efeito cardiovascular do álcool. Estudos futuros devem incluir a medição de outras variáveis, como pressão arterial ambulatorial 24 horas, variabilidade da frequência cardíaca, parâmetros cardiovasculares influenciados pelo sistema nervoso simpático e podem ser usados para prever um risco aumentado de doença cardiovascular.

\section{CONCLUSÃO}

Jovens adultos e bebedores compulsivos de bebida alcoólica apresentam mais elevada PPcentral quando comparado aos abstinentes. Os sujeitos bebedores compulsivos e moderados tendem a uma maior rigidez arterial pela análise da velocidade de onda de pulso comparado aos abstinentes. Os bebedores compulsivos classificados com ruim qualidade do sono apresentam maior PPcentral quando comparados aos bebedores moderados e abstinentes também com ruim qualidade do sono, ou seja, quanto maior o consumo de bebida alcoólica maior serão os distúrbios do sono e a PPcentral e, consequentemente, maior o risco de mortalidade por doença cardiovascular.

\section{AGRADECIMENTOS}

Agradecemos ao Prof. Shane A. Phillips, pela confiança em nós depositada para a análise dos dados de seu projeto de pesquisa.

\section{REFERÊNCIAS}

CLEMENT, Denis L. et al. Prognostic value of ambulatory blood-pressure recordings in patients with treated hypertension. New England Journal of Medicine, v. 348, n. 24, p. 2407-2415, 2003.

DIAZ, Keith M. et al. Visit-to-visit and 24-h blood pressure variability: association with endothelial and smooth muscle function in African Americans. Journal of human hypertension, v. 27, n. 11, p. 671-677, 2013. 
DOLAN, Eamon et al. Superiority of ambulatory over clinic blood pressure measurement in predicting mortality: the Dublin outcome study. Hypertension, v. 46, n. 1, p. 156-161, 2005.

GOSLAWSKI, Melissa et al. Binge drinking impairs vascular function in young adults. Journal of the American College of Cardiology, v. 62, n. 3, p. 201-207, 2013.

HASLER, Brant P.; SOEHNER, Adriane M.; CLARK, Duncan B. Sleep and circadian contributions to adolescent alcohol use disorder. Alcohol, v. 49, n. 4, p. 377-387, 2015.

HWANG, Chueh-Lung et al. The effects of repeated binge drinking on arterial stiffness and urinary norepinephrine levels in young adults. Journal of hypertension, v. 38, n. 1, p. 111-117, 2019.

MUNDT, Marlon P.; ZAKLETSKAIA, Larissa I.; FLEMING, Michael F. Extreme college drinking and alcohol-related injury risk. Alcoholism: Clinical and Experimental Research, v. 33, n. 9, p. 1532-1538, 2009.

JOHNSTON, Lloyd D. et al. Monitoring the Future national survey results on drug use, 1975-2012: College students and adults ages 19-50. Vol. 2. Ann Arbor: Institute for Social Research, The University of Michigan, 2013.

KENNEY, Shannon R. et al. Global sleep quality as a moderator of alcohol consumption and consequences in college students. Addictive behaviors, v. 37, n. 4, p. 507-512, 2012.

KENNEY, Shannon R. et al. Mental health, sleep quality, drinking motives, and alcohol-related consequences: a path-analytic model. Journal of Studies on Alcohol and Drugs, v. 74, n. 6, p. 841-851, 2013.

MENDES-PINTO, Daniel; DA GLÓRIA RODRIGUES-MACHADO, Maria. Aplicabilidade dos marcadores de rigidez arterial na doença arterial periférica. Jornal Vascular Brasileiro, v. 18, p. 0-0, 2019.

MEZICK, Elizabeth J. et al. Sleep duration and cardiovascular responses to stress in undergraduate men. Psychophysiology, v. 51, n. 1, p. 88-96, 2014.

MOON, Jeonggeun et al. Casual alcohol consumption is associated with less subclinical cardiovascular organ damage in Koreans: a cross-sectional study. BMC public health, v. 18, n. 1, p. 1091, 2018.

MS - Ministério da Saúde. Uma análise da situação de saúde e das doenças e agravos crônicos: desafios e $\begin{array}{lllll}\text { perspectivas. } & \text { Saúde } & \text { Brasil, } & \text { Disponível }\end{array}$ <http://bvsms.saude.gov.br/bvs/publicacoes/saude_brasil_2018_analise_situacao_saude_doencas_agravos_cr onicos_desafios_perspectivas.pdf>. Acesso em: 10 de março de 2020.

MUKAMAL, Kenneth J. et al. Binge drinking and mortality after acute myocardial infarction. Circulation, v. 112, n. 25, p. 3839-3845, 2005.

MUSINI, Vijaya M.; WRIGHT, James M. Factors affecting blood pressure variability: lessons learned from two systematic reviews of randomized controlled trials. PLoS One, v. 4, n. 5, 2009.

OPAS/OMS - Organização Pan-Americana da Saúde/Organização Mundial da Saúde. Doenças cardiovasculares: Doenças cardiovasculares, $2017 . \quad$ Disponível em: <https://www.paho.org/bra/index.php?option=com_content\&view=article\&id=5253:doencascardiovasculares\&ltemid=1096>. Acesso em: 10 de março de 2020.

PARATI, Gianfranco; LANTELME, Pierre. Blood pressure variability, target organ damage and cardiovascular events. Journal of hypertension, v. 20, n. 9, p. 1725-1729, 2002.

PNS/IBGE - Pesquisa Nacional de Saúde/Instituto Brasileiro de Geografia e Estatística. Pesquisa Nacional de Saúde 2013: Percepção do estado de saúde, estilos de vida e doenças crônicas. Disponível em: <https://www.ibge.gov.br/home/estatistica/populacao/pns/2013/default.shtm>. Acesso em: 10 de março de 2020. 
REHM, J.; SHIELD, K. Global alcohol-attributable deaths from cancer, liver cirrhosis, and injury in 2010. Alcohol Research: Current Reviews. v. 35, n. 2, p. 174-183, 2013.

SUNDELL, Laura et al. Increased stroke risk is related to a binge drinking habit. Stroke, v. 39, n. 12, p. 3179-3184, 2008.

WECHSLER, $\mathrm{H}$. et al. Trends in college binge drinking during a period of increased prevention efforts. Findings from 4 Harvard School of Public Health College Alcohol Study surveys: 1993-2001. Journal of American College Health, v. 50 , p. $203-217,2002$

WILKINSON, Amanda N. et al. Effects of binge alcohol consumption on sleep and inflammation in healthy volunteers. Journal of International Medical Research, v. 46, n. 9, p. 3938-3947, 2018.

YAYA, Sanni et al. Alcohol consumption and sleep deprivation among Ghanaian adults: Ghana Demographic and Health Survey. PeerJ, v. 6, p. e5750, 2018. 